\title{
VOCI DI ORIGINE RUSSA IN ITALIANO
}

\section{Premessa. Incidenza quantitativa dei russismi nel lessico italiano}

I recenti progressi della lessicografia e la crescente diffusione dei repertori informatizzati permettono di basare su dati oggettivabili la valutazione dei vari strati che compongono il lessico di una determinata lingua. Chi in particolare volesse sottoporre a campionamento statistico i materiali di provenienza esogena non avrebbe difficoltà a estrarre i valori numerici che esprimono l'incidenza dei diversi apporti lessicali e a produrre calcoli dall'elevato potere informativo: lo strumento che meglio si presta a tale operazione è sicuramente il GRADIT, da cui è stato estratto il Dizionario delle parole straniere nella lingua italiana. Progetto e direzione scientifica: Tullio De Mauro e Marco Mancini, Milano, Garzanti Linguistica, 2001.

Se ad esempio prendiamo a riferimento la tav. 4 riportata nel VI e ultimo volume del GRADIT (p. 1170), abbiamo agio a notare che ad una matrice russa possiamo assegnare 86 cosiddetti 'esotismi in senso stretto' (da intendersi come "parole d'origine esterna all'italiano non adattate dal punto di vista fonomorfologico e non integrate nel sistema derivazionale italiano" ibidem)", 166 unità lessicali adattate e 92 'esotismi non adattati di ambito tecnico e specialistico': tali dati collocano il russo al settimo posto nella classifica degli apporti esogeni al lessico italiano, dopo l'inglese, il francese, lo spagnolo, il tedesco, l'arabo, il provenzale ma prima, per fare solo qualche esempio, del portoghese e del longobardo.

Un'altra istruttiva ricerca concerne l'articolato e complesso ordinamento delle voci in funzione di tredici cosiddette "marche d'uso" ( 0 , all'inglese, labels), che permettono di caratterizzare le espressioni secondo la loro appartenenza regionale, settoriale, di registro e secondo la frequenza d'impiego. Prima di analizzarne le potenzialità, è bene chiarire cosa si intenda con questa locuzione: si tratta in definitiva di un contrassegno "che identifica l'appartenenza dei lemmi o di loro accezioni al vocabolario di base (fondamentale, di alto uso o di alta disponibilità, comune, letterario, di basso uso o obsoleto) o a uno dei linguaggi tecnico-specialistici e ne indica, all'occorrenza la natura di esotismo non adattato".

\section{Lineamenti di storia della ricerca}

Ma probabilmente la ricognizione dei russismi potrebbe permetterci inferenze ancora più rilevanti se lo scavo documentario delle fonti da cui discendono i dati quantitativi fosse stato più esteso e approfondito e se la ricerca in quest'ambito fosse stata più sistematica. 
In realtà è ben noto che lo spazio destinato agli slavismi nell'economia delle trattazioni manualistiche di storia linguistica italiana e in lessicografia, quanto meno fino all'avvio degli anni Ottanta, era piuttosto modesto specie se rapportato alla costante attenzione assicurata ai francesismi e agli anglicismi; forse in una condizione analoga di sottostima si pongono i tedeschismi del XIX e del XX secolo che in realtà sono molto pervasivi del lessico intellettuale e scientifico e in particolare della terminologia linguistica (cfr. SoRBA 2002).

Unica eccezione ad un interesse così tiepido era rappresentata dal Dizionario moderno di A. PANZINI, che fin dal 1905 raccoglieva una ricca serie di lemmi, in modo comunque frammentario e orientato da una parte a caratterizzare in chiave esotica i russismi di epoca zarista e dall'altra a sovraccaricare i neologismi postrivoluzionari (accolti, a partire dall'edizione del 1918) di implicazioni valutative sociopolitiche che facevano perdere di vista la pertinenza linguistica di tali influenze.

Le Appendici curate da B. Mrgliorini $(1942,1950,1963)$ sono sicuramente più sobrie ed hanno avuto anche il merito di aggiornare il quadro neologico alle espressioni del secondo dopoguerra, filtrate nella maggior parte dei casi attraverso intermediazioni occidentali che oscurano l'ascendenza russa relegandola sul piano dell'antefatto remoto in ogni caso non rendendo ragione dell'esistenza di un ben preciso e identificabile flusso di lessico e fraseologia legati all'esperienza storica e politica dell'Unione Sovietica ${ }^{1}$. Un caso emblematico è riconoscibile nella categoria politica della deviazione e nei suoi derivati deviazionismo e -ista, diligentemente registrati dal Migliorini ma non posti in connessione con gli archetipi russi $u k l o n, u k l o-$ nizm e -ist dei quali rappresentano calchi semantici; la serie espressiva va in effetti ricondotta a un modulo locutivo polemico di cui prima Lenin, poi Stalin e infine le leaderships a lui succedutesi al Cremlino si servivano per bollare gli avversari politici. Non diversa è la genesi del tipo derivativo rappresentato dalle formazioni avventurismo e avventurista che, lungi dall'essere estratte per trafila ereditaria da avventu$r a$, meglio si chiariscono come prestiti di un altro dispositivo formulare del vocabolario bolscevico, che anche in questo caso prende di mira una condotta politica giudicata inaccettabile e cioè la 'fuga in avanti', la scelta massimalista e irresponsabile di chi non si fa carico di una visione generale corrispondente agli effettivi interessi della collettività (per una testimonianza recente cfr. "Il Gazzettino" 9 ottobre 1998, p. 3 tit.: Galante: "Fausto, scelta avventurista").

Continuando questo breve profilo di storia della ricerca sull'elemento russo in italiano, possiamo individuare una soglia cronologica di riferimento che coincide con il 1982, anno di pubblicazione del primo dei due volumi che si devono a Giorgio Maria Nicolai. Non si trattava di un repertorio in senso proprio ma di una sequenza di voci monografiche attinenti, come puntualizzava l'A., ai realia, ossia all'ambito delle istituzioni, delle peculiarità etniche e di costume del mondo russo di epoca

1 Sulle mediazioni esercitate da lingue terze sui russismi in italiano cfr. "IncLing." 15 (1992), pp. 107-124. 
zarista, da zar a rublo, dalla vodka alla steppa, passate in rassegna secondo una impostazione orientata a parificare il russismo ad esotismo (spesso mediato da una ricezione letteraria). Ma, accanto ai russismi storici, Nicolai accoglieva una pattuglia di voci riconducibili a tutt'altra tipologia, quella dei s ovie t is $\mathrm{m} i$; a scorrere quella lista, mi resi conto della portata interlinguistica di tali innovazioni, dello spazio che si erano guadagnate nel lessico italiano, entro il quale occupano a mio parere una posizione non periferica e comunque non limitata alla lingua speciale della politica. Da qui l'impulso alla realizzazione di un contributo (ORIOLES 1984) che sviscerasse questa specifica tipologia, dedicando particolare enfasi da una parte alle forme di influenza non esplicite, ossia ai calchi strutturali o di significato e ai prestiti 'camuffati' (mimetizzati cioè da estensione semantica di forme ereditarie), e dall'altra agli slittamenti metaforici, all'uso figurato di russismi che sull'onda di eventi politici, di una percezione forte e ideologizzata del loro impatto, superavano i confini del tecnicismo per dotarsi di valenze e connotazioni estranee alla lingua di partenza. Non era in effetti facile cogliere in unità lessicali ed espressioni come autocritica, cinghia di trasmissione, coesistenza pacifica, disgelo, purga la sollecitazione proveniente dalle corrispondenti forme in lingua russa.

$\mathrm{Ma}$ anche formazioni che evidenziavano la loro provenienza, prestiti come pogrom, trojka, intelligencija, niet superavano indubbiamente i limiti e lo statuto dell'esotismo nella misura in cui erano piegate ad esigenze espressive proprie della lingua d'arrivo, che le utilizzava per rimediare a lacune delle proprie strutture semantiche: la trojka, ad esempio, in origine nome di una vettura da traino, e poi estensivamente designazione di un triumvirato di esponenti politici sovietici, si prestò ad indicare un qualsiasi gruppo di tre leaders che conducessero una azione politica concertata, come si verificò più volte per i titolari dei tre ministeri del bilancio, delle finanze e del tesoro ai quali era demandata dal governo italiano una sorta di direzione collegiale dell'economia (La Malfa, Colombo, Giolitti).

Questo era in estrema sintesi il senso di un lavoro (ORIOLES, Tipologie) che, a volerlo storicizzare, si inscrive innanzitutto in un rifiorire della lessicografia italiana che si dota di nuovi strumenti; vorrei qui ricordare che gli anni dal 1979 al 1987 sono quelli della prima edizione del DELI di Manlio Cortelazzo e Paolo Zolli (oggi continuato nel Nuovo DELI) e dell'avvio dell'itinerario del LEI, ideato e coordinato da Max Pfister. A Tipologie avrebbero fatto seguito vari interventi della scuola che si raccoglie attorno a "Lingua Nostra" (FANFANI 1987 e 1995; TESI 1993) e poi i pregevoli studi dedicati da Marco Mancini alla categoria dell'esotismo, che gettano nuova luce sulle vie di penetrazione dei russismi anteriori al Novecento (MANCINI 1992 e 1994); anche la storiografia umanistica si apriva alla considerazione del russismo retrodatando in modo consistente la prima segnalazione di parecchie voci attestate nelle 
relazioni di viaggio raccolte dal Ramusio (Giovio, Herberstein, Guagnini ecc.) e poi, sul finire del XVI sec., nella Moscovia di Antonio Possevino².

Una reviviscenza di studi sull'apporto lessicale russosovietico è legata al gorbaciovismo, ossia con l'insieme compatto di espressioni riferibili all'effimera stagione riformatrice di Michail Gorbacëv (compendiata da espressioni quali glasnost', perestrojka) che poi prelude alla rovina del comunismo scandita dalla caduta del muro di Berlino (1989) e dallo scioglimento dell'U.R.S.S. (1991): colgono questa fase di trapasso gli studi della LASORSA SIEDINA (1990a e 1990b) sul linguaggio politico gorbacioviano e la seconda monografia di NICOLAI 1994 (Viaggio lessicale; se ne veda la recensione in "Incontri Linguistici") ${ }^{3}$; riflesso di un filone di studi coltivato su scala internazionale (CORTEN 1992 ecc.).

\section{Riflessi semantici}

Esaurita la parte retrospettiva del mio contributo, è giunto il momento di ritagliare una sezione del vastissimo temario che ruota attorno al quadro generale dei russismi e dei sovietismi in italiano. La parte indubbiamente più significativa dell'interferenza russa in italiano è legata alla semantica, ed in particolare, come ho prima accennato, al recupero di prestiti e calchi in accezioni traslate e a volte persino contrapposte a quelle assunte dal termine nella lingua modello. Ora, che una forma alloglotta venga piegata ad usi estensivi esulerebbe dalla fase vera e propria del contatto per rientrare tra i prevedibili sviluppi che scandiscono le vicende posteriori alla mutuazione, tutte interne alle strutture lessicali della lingua replica. Si tratta di quella che Filipović, ha definito secondary adaptation per distinguerla dalla primary adaptation, che ricopre invece le alterazioni intervenute all'atto stesso della mutuazione (cfr. a titolo indicativo FILIPOVIĆ 1977, ma si tratta di un principio esplicativo su cui l'Autore è tornato a più riprese). E tuttavia la rilevanza di tale fenomeno è tale da non permetterci di escludere, in nome di un rigore formale, un materiale così rilevante.

$\mathrm{Nel}$ prendere in esame questa fattispecie ${ }^{4}$ sarà utile distinguere innanzitutto tra russismi figurati di epoca zarista e traslazioni che operino su sovietismi, ossia su unità lessicali che recano impresso in sé il 'marchio' dell'esperienza istituzionale sovietica (e dunque riferibili al periodo compreso fra il 1917 e il 1991, fra la data della Rivoluzione d'ottobre e l'anno che vide dissolversi l'U.R.S.S.).

2 Sulla personalità di Possevino si veda L'alterità linguistica della Moscovia nei Commentari di Antonio Possevino, in Una pastorale della comunicazione. Italia, Ungheria, America e Cina: l'azione dei Gesuiti dalla fondazione allo scioglimento dell'Ordine, a cura di D. PoLI, Atti del Convegno di Studi (Roma - Macerata, 24-26 ottobre 1996), Roma 2002, pp. 477-489.

3 Il riferimento è a "Incontri Linguistici" 18 (1995) [1996], pp. 211-228. Nelle more di una edizione accresciuta di Tipologie, faro riferimento d'ora innanzi a una serie di lavori apparsi dopo il 1984; le relative sigle vengono sciolte nei riferimenti bibliografici.

4 Espressamente trattata in ORIOLES 1993 ma ripresa anche in ORIOLES 2000. 
Per ciò che concerne gli usi estensivi applicati a russismi storici basti qui ricordare i casi risaputi di trojka, ukase, pogrom, zar, zarina ecc.; quanto ai sovietismi menzionerò i casi tuttora vitali di nomenklatura, niet, cinghia di trasmissione ecc..

Nomenklatura in particolare è un sovietismo di età brezneviana, invalso - dopo la denuncia di Voslensky ${ }^{5}$ - per indicare la ristretta classe di esponenti dell'apparato del partito ammessi a godere benefici (la vacanza, la dacia, l'accesso a beni di consumo) dai quali erano esclusi i comuni cittadini, ed è sopravvissuto al declino dell'U.R.S.S. conquistandosi un territorio stabile anche dopo che sono venute meno le condizioni storiche che ne segnarono la fortuna; oggi, in effetti, nomenklatura indica ogni tipo di "oligarchia, casta di privilegiati".

Un singolare risvolto della semantica dei russismi di età sovietica è visibile in una pattuglia di espressioni che sono il correlato linguistico della frattura in due blocchi del mondo politico e della conseguente scissione ideologica dei parlanti: agitazione, agitatore; stacanovismo -ista; socialismo reale; cosmopolita e cosmopolitismo; dissidente sono l'esemplificazione di questa tipologia che fa di una stessa espressione volta per volta un 'sovietismo' ovvero, secondo una proposta terminologica che ho formulato in ORIOLES 1994, un 'antisovietismo'.

Il termine più calzante da prendere a riferimento può essere scorto nell'espressione stachanovskoe dviženie ("movimento stacanovista") che, da idea-forza della propaganda sovietica a favore dell'intensificazione dei ritmi produttivi, scade, una volta fatto proprio dai circoli non comunisti dell'Occidente, a stereotipo caricaturale di ogni forma di zelo eccessivo nel lavoro. Originariamente avvertito come 'sovietismo', il sintagma russo, diffuso in Italia attraverso canali estranei alla sinistra, finisce appunto col trasformarsi in una sorta di 'antisovietismo'.

E' ben noto che alcuni moduli locutivi del lessico politico si prestano ad essere semanticamente anfibolici, bifronti, suscettibili dunque di essere interpretati in un senso $o$ in quello antinomico a seconda della propensione culturale e ideologica di chi ne fa uso.

Si tratta di un insieme di unità lessicali apparentemente informative ma che in realtà traggono una forte carica valutativa dal contesto sociale e istituzionale in cui si ritrovano: rispondono a questa caratteristica ad esempio "democrazia", "borghese", "conservatore", "rivoluzionario", "comunista", "monarchico", "revisionista", "formalista".

Il fenomeno ha conosciuto comprensibilmente una notevole incidenza nei rapporti tra paesi comunisti e mondo occidentale, in quanto i rispettivi linguaggi politici condividevano una base lessicale formalmente convergente, ispirata al comune patrimonio espressivo; ma le nozioni da essi designate catalizzavano o adesione fideistica o feroce opposizione caricandosi, a seconda dei casi, di connotazione positiva o negativa.

5 Si fa riferimento a M. VosLensKY, Nomenklatura. La classe dominante in Unione Sovietica, Milano 1980. 
Tipologie collaterali, ma non prive di riflessi, cui vorrei dedicare un breve cenno sono quelle dei sovietismi che 'smarriscono' le loro primitive radici culturali e per converso dei russismi storici che riguadagnano centralità e vedono potenziata la loro originaria delimitabile valenza. Diversamente dai sovietismi 'canonici', che in qualche maniera mantengono una percepibile connessione con il contesto storico-culturale che li ha generati, le espressioni del primo gruppo hanno conosciuto uno sviluppo semantico imprevedibile in rapporto al modello ispiratore: tra esse si possono annoverare casi come arcipelago, autogestione, avventurismo, collettivo, disfattismo, partigiano $^{6}$, quadri, rivoluzione culturale, tutte voci che, pur in ultima analisi riconducibili ad archetipi russosovietici, sono l'epilogo di trafile complesse (intermediazioni di terze lingue, calco attraverso espressioni di familiare aspetto latino o romanzo, profonde rielaborazioni culturali e vere e proprie distorsioni semantiche) che hanno reso irriconoscibile il loro status di 'sovietismo'. Altrettanto interessante il caso dei russismi di epoca zarista tornati in auge nel XX secolo: fermo restando che in questa sede dobbiamo limitarci ad una esemplificazione orientativa, vale la pena ripercorrere le vicende di boiardo, variante di boiaro, designazione dell'aristocrazia che circondava lo zar. È interessante far notare che, a seconda della suffissazione, il medesimo antefatto russo è andato incontro a una distinta caratterizzazione semantica: mentre il tipo boiaro si configura come un semplice russismo storico $^{7}$, la variante munita del suffisso francesizzante in ardo ha sviluppato una marcata connotazione peggiorativa che l'ha resa utilizzabile anche in contesti avulsi da ogni riferimento al mondo russo. In particolare, nella pubblicistica italiana degli anni Settanta del XX sec., si è andata creando l'identificazione dei boiardi con i potenti e spregiudicati managers delle grandi imprese pubbliche, capaci di esercitare, nel bene e nel male, una influenza sugli equilibri economici ed anche politici del Paese; la voce sarebbe rimasta confinata nel novero delle voci storiche se non avesse conosciuto una inopinata ripresa e rivitalizzazione intorno al 1977 (in coincidenza con la pubblicazione del volume Razza padrona, di Eugenio Scalfari) ${ }^{8}$.

6 Per questa voce basti qui il rinvio all'ampia messa a punto di R. Tesi (Nuove proposte per 'partigiano', "Lingua Nostra" 54, 1993, pp. 73-84), che riprende e perfeziona l'impostazione della voce compresa in Orioles, Tipologie.

7 Per l'ipotesi di una mediazione polacca o ceca del tipo boiaro (il russo bojarin non può esserne la fonte diretta) cfr. W. DAHMEN - K. HENGST - J. KRAMER, Sur la voie d'un Etymologicum Graeco-Slavo-Romanicum, in G. HrLrY (ed.), Actes du XXème Congrès International de Linguistique et Philologie Romanes (Université de Zurich, 6-11 avril 1992), Tome IV, Tübingen-Basel, 1993, pp. $401-417$ (a p. 410 n. 13).

8

Nicolai 1984 s.v. boiaro rinvia ad una eloquente testimonianza ("Epoca" 26 ottobre 1977: intervista di Domenico Bartoli a Giuseppe Petrilli, Presidente dell'IRI) della quale vale la pena riportare il passaggio più significativo: "Vi chiamano razza padrona, borghesia di Stato, b o i a r d i. Boiardi, cioè grandi e potenti aristocratici come quelli che, nella vecchia Russia, Pietro il Grande domò con durezza ... Boiardo, una parolaccia, un incrocio fra boia e bastardi" Prima ancora che si stabilisse la sistematica correlazione dei boiardi con i dirigenti degli enti pubblici, il termine aveva conosciuto una prima forma di riattivazione con il titolo del film di Sergej Eisenštein, La congiura dei boiardi (originale russo: Bojarsky zagovor), diffuso nel 1958. Devo l'informazione a Giorgio Graffi, che qui ringrazio. 


\section{Effetti strutturali dell'influenza russa in italiano?}

Accanto all'indiscussa influenza che si esercita in termini di aggiunte all'inventario lessicale ovvero di impatto semantico (vedi $\S 3$ ), sarà da riconsiderare l'intera materia dell'apporto linguistico russo per individuare eventuali ricadute che investano le strutture formali.

Naturalmente siamo ben lontani dal vistoso rimodellamento legato all'influenza inglese, che amplia i limiti distribuzionali delle unità fonemiche, innova nella sequenza determinante-determinato, alimenta le risorse composizionali creando i presupposti di una vera e propria frattura tipologica, ma una qualche incidenza sull'italiano, così come sull'insieme delle lingue europee occidentali, va riconosciuta anche al russo.

Escluso il livello fonologico, cominciamo a soffermarci sulla cosiddetta 'induzione di morfemi' verificando ad esempio se anche in italiano possano ravvisarsi casi comparabili con l'inglese, specialmente l'inglese d'America, che dal russo ha acquisito la familiarità col suffisso -nik che da sputnik, famosa designazione dei satelliti artificiali, ha potuto essere estrapolato e applicato a termini indigeni: basti qui ricordare il caso di beatnik, impensabile senza la sollecitazione di sputnik. La fortuna italiana del suffisso non è pari a quella goduta in ambito inglese, proprio perché non è stata sostenuta dal preesistente -nik dello yiddisch, ma ciononostante un qualche effetto lo ha prodotto, se è vero che il derivato apparatčik, con cui si evoca l'uomo di apparato, il funzionario addetto alla macchina di partito, spesso viene frainteso come apparatnik. Un'altra nicchia nella quale elementi formativi di ascendenza russa manifestano produttività è quella guadagnata dal suffisso -acija riconoscibile ad esempio in normalicacija, tradotto con normalizzazione; qui il parlante che abbia un certo livello di competenza bilingue coglie la sistematica corrispondenza interlinguistica che si instaura tra i due morfemi ed è quindi in grado, quanto meno potenzialmente, di sfruttare il forte potere evocativo del suffisso per applicarlo a basi lessicali native: $\mathrm{mi}$ è capitato che una delle numerose commissioni di cui faccio parte andasse in trasferta in una sede staccata a visionare dei locali; al che un collega fotografò la rituale solennità dell'evento dicendo lapidariamente: "Ecco è arrivata la delegacija". Per quanto si tratti di fatti periferici, nell'uno e nell'altro caso si può parlare di morfemi muniti di valenza stilistica, ovvero, per usare un costrutto fatto valere da Meo Zilio, di morfostilemi.

Per restare nell'ambito degli elementi formativi ricavati da materiale lessicale russo, un cenno lo merita l'elemento -strojka ricavato per decurtazione da perestroj$k a$; e qui bisogna dare atto al collega Sgroi di averne per primo rilevato, in un puntuale intervento del 1987, il riutilizzo in formazioni ispirate a ludismo verbale quali catastrojka nelle quali -strojka funge da suffissoide.

Un ruolo significativo, e fin qui poco esplorato, va poi riconosciuto a una tendenza strutturale che tanta parte aveva nelle abitudini formulari del linguaggio politico russosovietico: alludo alla proliferazione di abbreviazioni e acronimi, che finirono 
per costituire un vero e proprio modulo stilistico. In effetti l'ipotesi, affacciata da più parti, è suggestiva: le diverse tipologie di sigla (inizialismi, sigle sillabiche, decurtazioni di vario genere) che costituiscono un modulo prediletto dal russo postrivoluzionario (cfr. tra gli altri COMRIE 1996) sono il risultato di un trend strutturale condiviso dalle lingue ufficiali dei paesi retti da regimi totalitari, come la Germania nazista e l'Italia fascista, le cui istituzioni venivano frequentemente designate da strutture accorciate: la GPU e la Ceka tristemente famose, il Gosplan, il Gu-lag, il Komintern, la Tass, l'Aeroflot, il diamat. Considerato che in quegli stessi anni anche tedesco e italiano facevano ricorso a forme strutturalmente affini (quali Gestapo, Minculpop, Confindustria), è plausibile una primogenitura russa del procedimento? Il tema andrà ripreso: è di prossima pubblicazione un rifacimento di Tipologie, nel quale sarà dedicato più spazio alle ripercussioni sistemiche dell'influenza linguistica russa.

\section{Conclusioni}

Volendo estrarre un giudizio conclusivo dai materiali proposti in questo lavoro, se si volesse diagnosticare lo stato di salute dei russismi in italiano al di là della pura e semplice incidenza quantitativa, non si sarebbe lontani dal vero ad affermare che le voci collegate con il mondo russo si sono conquistate un ben preciso segmento all'interno delle strutture lessicali italiane anche per il peculiare spazio comunicativo ad esse correlato. E' vero: si deve prendere atto del declino di un consistente settore di espressioni russosovietiche, di quelle cioè più strettamente legate alle vicende ideologiche e istituzionali; per converso reggono bene all'urto i sovietismi sottratti al loro ambito d'origine e inseriti nel circuito comunicativo comune, diventati elementi formulari, capaci di rispondere a precise esigenze espressive e stilistiche. E dunque, se sono ormai relegate alla periferia del sistema formazioni quali cellula, centralismo democratico, fattoria collettiva, Armata rossa, vigilanza rivoluzionaria, resistono saldamente nell'uso tipi lessicali come normalizzazione, nomenklatura, disgelo, autocritica, cinghia di trasmissione e trasparenza. Una inopinata fortuna arride in particolare a quest'ultima espressione che, sganciata al contesto storico-politico che l'aveva generata, è stata persino inserita nel 1992 in una legge che regola i rapporti tra cittadini e istituti di credito: singolare destino di quello che quindici anni fa era un sovietismo caratterizzante di una svolta considerata promettente. 


\section{Abbreviazioni correnti}

DELI = M. CORTELAZZo - P. ZOLLI, Dizionario etimologico della lingua italiana, 5 voll. Bologna 1979-1988.

GRADIT = Grande Dizionario Italiano dell'Uso, ideato e diretto da Tullio De Mauro, con la collaborazione di

Giulio C. Lepschy e Edoardo Sanguineti, 6 voll., Torino 1999 (con allegato un CD edito nel 2000).

LEI = Lessico etimologico italiano, diretto da Max Pfister, Wiesbaden 1979-.

Nuovo DELI (DELIN) = Dizionario etimologico della lingua italiana, seconda edizione a cura di Manlio CORTELazzo e Michele A. CoRTELAzzo, Bologna 1999.

LRL = Lexikon der Romanistischen Linguistik, hrsg. von G. HoLTus - M. METZELTIN - Ch. SCHMITT.

\section{Riferimenti bibliografici}

CORTEN 1992 = Irina H. CORTEN, Vocabulary of Soviet Society and Culture. A Selected Guide to Russian words, Idioms, and Expressions of the Post-Stalin Era, 1953-1991, London 1992.

FANFANI $1987=$ M. L. FANFANI, Russismi politici novecenteschi: a proposito di un libro di Vincenzo Orioles, "Lingua Nostra" 48 (1987), pp. 59-84.

FANFANI $1994=$ M. L. FANFANI, recensione di M. MANCINI, L'esotismo nel lessico italiano, in "Lingua Nostra" 55 (1994), pp. 121-126.

FELLERER 1998 = J. FELLERER, voce n. 467 Slavisch und Romanisch/Les slave et les langues romanes, in LRL, Band/Volume VII, Kontakt, Migration und Kunstsprachen. Kontrastivität und Typologie/Analyses contrastives, classification et typologie des langues romanes, Tübingen 1998, pp. 184-230.

FiLIPović 1977 = R. FiLIPović, Primary and Secondary Adaptation of Loan-Words, "Wiener Slavistisches Jahrbuch" 23 (1977), pp. 116-125.

GuSMANI 1986 = R. GuSMANI, Saggi sullinterferenza linguistica, Firenze $1986^{2}$ (rist. 1993).

LASORSA SIEDINA 1990a = C. LASORSA SIEDINA, Il discorso politico di M. S. Gorbačev in Linguistica selecta I (Pubblicazioni del Dipartimento di Scienze del Linguaggio dell'Università di Roma "La Sapienza": Ricerche/7), Roma 1990, pp. 33-84; apparso anche in "Rassegna sovietica" III (maggio-giugno 1989), pp. 128-148.

LASORSA SIEDINA $1990 \mathrm{~b}=$ C. LASORSA SIEDINA, Il linguaggio giornalistico: europeismi nel russo e russismi in italiano (1986-1989), in Linguistica selecta cit., pp. 85-102.

MaNCINI 1992 = M. Mancinl, L'esotismo nel lessico italiano, Viterbo, Università degli Studi della Tuscia, Istituto di Studi Romanzi ("Biblioteca di Ricerche linguistiche e filologiche" 32), 1992.

MANCINI $1994=$ M. MANCINI, Voci orientali ed esotiche nella lingua italiana. $\$ 5.1$ Sovietismi, in L. SERIANNI - P. TRIFONE (a cura di), Storia della lingua italiana, III. Le altre lingue, Torino 1994, pp. 875-878.

NICOLAI 1982 = G. M. NICOLAI, Le parole russe, Roma 1982.

Nicola 1994 = G. M. Nicolal, Viaggio lessicale nel paese dei Soviet. Da Lénin a Gorbacëv, Roma ("Biblioteca di Cultura" 489), 1994.

ORIOLES Tipologie $=$ V. ORIOLES, Su alcune tipologie di russismi in italiano, Udine, Università degli Studi, 1984.

ORIOLES $1993=$ V. ORIOLES, Russismi di senso figurato in italiano, "Quaderni dell'Istituto di Glottologia dell'Università «D'Annunzio» di Chieti” 5 (1993), pp. 103-124.

ORIOLES 1994 = V. ORIOLES, Sovietismi ed antisovietismi, in Miscellanea di studi linguistici in onore di Walter Belardi, a cura di P. CIPRIANo, P. Di GIovine, M. MANCINI, II, Roma 1994, pp. 667-673, ripreso con aggiornamenti in Percorsi di parole, Roma 2002, pp. 115-122.

SGRor 1987 = S. C. SGRoI, Perestrojka per tutti, "La Sicilia" 12 dic. 1987, riproposto in Bada come parli. Cronachette e storie di parole, Torino 1995, pp. 49-51.

SORBA 2002 = G. SORBA, I tedeschismi nella terminologia linguistica, "Plurilinguismo. Contatti di lingue e di culture" 7 (2000) [2002], pp. 187-237.

TESI 1993 = R. TESI, Nuove proposte per partigiano, "Lingua Nostra" 54 (1993), pp. 73-84. 


\section{BESEDE RUSKEGA IZVORA V ITALIJANŠČINI}

Prispevek skuša pregledno predstaviti dosedanje raziskave o jezikovnih vplivih ruščine na italijanščino; pri tem se ugotavlja, da ti vplivi še niso bili zadostno osvetljeni. Tako v carski dobi kot v času od Oktobrske revolucije do razkroja Sovjetske zveze (1917-1991) je bilo v italijanščino sprejetih dokaj ruskih besednih prvin, prevzetih neposredno iz ruščine ali posredno, preko drugih jezikov. Pomemben napredek v leksikografiji, tudi tehnične pridobitve, dovoljujejo ugotovitev, da je za dotok tujega besedja v italijanščini ruski jezik na sedmem mestu, in sicer za angleščino, francoščino, španščino, nemščino, arabščino, provansalščino, a na primer pred portugalščino ali longobardščino. S̆tudija nadaljuje s tipološko klasifikacijo različnih oblik jezikovnega stika: tudi tu se pojavlja tista znana razdelitev med zgodovinskimi rusizmi ( $v$ glavnem za konkretne pojme) in t.i. sovjetizmi. Želi pa se opozoriti tudi na številne 'nevidne' izposojenke, torej na kalke.

Veliko prevzetih besed kaže pomenske spremembe, predvsem razširjanje pomena in pa rabo v prenesenem pomenu; ob koncu se prispevek dotika možnih strukturnih vplivov, tako zlasti morfemov pri številnih kraticah in na splošno pri besednih krajšavah. 